\title{
The relevance of family engagement in the implementation of the Picture Exchange Communication System (PECS) in children with Autism Spectrum Disorder
}

\author{
Dayane Oliveira Olivatti ${ }^{1}$ \\ https://orcid.org/0000-0001-8426-5564 \\ Mayara K Sugahara ${ }^{1}$ \\ https://orcid.org/0000-0002-2970-0911 \\ Simoni Camilo ${ }^{1}$ \\ https://orcid.org/0000-0002-8204-444X \\ Jacy Perissinoto ${ }^{1}$ \\ https://orcid.org/0000-0002-0287-9296 \\ Ana Carina Tamanaha ${ }^{1}$ \\ https://orcid.org/0000-0001-9915-6299
}

Universidade Federal de São Paulo - UNIFESP, Departamento de Fonoaudiologia, São Paulo, São Paulo, Brasil.

Research support source: Conselho Nacional de Desenvolvimento Científico e Tecnológico - CNPq. (445979/2014-3) e (421937/2018-1)

Conflict of interests: Nonexistent

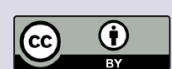

Received on: April 30, 2021

Accepted on: August 16, 2021

Mailing address:

Ana Carina Tamanaha

Rua Botucatu, 802, Vila Clementino

CEP: 04023-900 - São Paulo, São Paulo, Brasil

E-mail: anacarinatamanaha@gmail.com

\section{ABSTRACT}

Purpose: to analyze the relevance of family engagement in the implementation of the Picture Exchange Communication System (PECS) in children presented with Autism Spectrum Disorder (ASD).

Methods: a longitudinal study with a sample of 22 mother- child dyads with ASD; 17 boys and 5 girls, with a mean age of 7 years and 2 months. The Executor Skills Protocol was used. The PECS implementation program consisted of 24 therapy sessions with the active presence of mothers. The family's support was measured by the frequency of the sessions. Spearman's correlation test and a significance level of $0.05 \%$ were used.

Results: mothers performed most tasks correctly in the first three phases. There was a tendency to correlation between the executor's skills and the number of sessions in all phases of the program, and in the four initial phases, the indexes showed a statistical significance. Family compliance was $96 \%$.

Conclusion: family engagement was relevant for the appropriation of children to the PECS during the time of exposure, reaching phases of discrimination and construction of sentences with cards.

Keywords: Autism Spectrum Disorder; Language; Communication; Family; Speech, Language and Hearing Science 


\section{INTRODUCTION}

Autism Spectrum Disorder (ASD) is characterized by severe impairments in the areas of interaction and social communication and by a restricted and stereotyped repertoire of interests and activities ${ }^{1}$

A significant number of people affected by ASD cannot use oral communication, therefore, they need an alternative resource that allows them to initiate, sustain, and expand their dialogical situation, considering the disabilities of joint attention, deviant gaze, and the lack of intentionality ${ }^{2}$.

Picture Exchange Communication System (PECS) is currently one of the most widely used communication programs worldwide for people with ASD. This system is composed of pictures/symbols selected according to the lexical repertoire of each subject and involves the substitution of speech by a figure, encouraging thus the expression of needs and desires. The system is based on applied behavior analysis, so that specific stimulation and reinforcement strategies are used that will lead to independent communication. In addition, systematic correction procedures are used errors, which aim at learning even when the error occurs.

Implementation should be carried out by experienced speech therapists who should encourage the constant participation of different communicative partners and respect the following phases: In phase I (physical exchange: how to communicate), children are encouraged to use cards to request/show their desire for an object that is attractive to them. Phase II (distance and persistence), the objective is for the child to effectively understand the importance of using cards and continue to use them in any communicative situation. In phase III (discrimination between symbols), children are encouraged to select a target picture from several options. They must discriminate the cards and hand the most appropriate one to the communicative partner. At this phase, children can showcase their intentionality by the autonomous choice of their reinforcer. In phase IV (using phrases), users learn to build sentences with cards using action verbs (e.g. to want) and characteristics of the objects (e.g., color, size). In this stage, the functional vocabulary is considerably expanded. In phase V (answering "What do you want?"), children are encouraged to answer the question "What do you want?" with simple phrases in the cards. In phase VI (commenting), users answer questions such as "What do you see?"; "What are you listening to?" "What is this?", besides asking and spontaneously commenting on situations/events using simple phrases with the cards ${ }^{3}$.

Notably, the PECS implementation must be individually evaluated, assuring the involvement of all interlocutors ${ }^{2,3}$, since the family's engagement in any treatment ensures the expansion of therapeutic objectives in the household context, providing greater synchronicity, communicative and social contingency between children and their interlocutors. At the same time, it empowers the family, reducing its emotional overload $^{4-6}$.

The hypothesis of this study is that parental engagement is fundamental to ensure the success of the PECS implementation in children, since parents are the communicative partners who promote the systematic and daily use of the system.

Thus, this study aimed to analyze the relevance of family's engagement during the implementation of the PECS program in autistic children who are not verbal or with minimal verbalization. Also, its purpose was to analyze the effect of the PECS implementation on maternal overload and severity of ASD, in children.

\section{METHODS}

This is a longitudinal, experimental and qualitative study.

All parents or guardians were aware of the methodological procedures of the study and signed the informed consent form approved by the Research Ethics Committee of Universidade Federal de São Paulo - UNIFESP, Brazil (Opinion No. 1284/2017).

In total, 22 children composed the convenience sample, 17 boys (77\%) and five girls (23\%); aged between six and 12 years, they were attended and diagnosed with ASD by a multidisciplinary team, according to the diagnostic criteria of DSM-5 (The Diagnostic and Statistical Manual of Mental Disorders), between March 2016 and March 2019.

Regarding the speech extension obtained from the application of the Vocal Behavior Assessment ${ }^{4}, 19$ children (86\%) demonstrated non-verbal production (babble emission and/or vocalizations) and 3 children (14\%), minimal verbal production: emission of isolated words or juxtaposition (with no use of verbs), during the speech-language evaluation period.

Regarding the cognitive profile, the testing was carried out concurrently with the speech-language assessment and was carried out by the neuropsychologist team. The distribution of intellectual quotient 
values was concentrated in the lower range, with a mean score equal to $50.5(\mathrm{SD}=9.4)^{22}$.

All children were regularly enrolled in regular schools due to the Brazilian policy of school inclusion, on average for forty-five months $(S D=21.9)$ and had already been exposed to previous speech therapy intervention in different care services, for at least six months of duration, to guarantee that the communicative profile was characterized as non-verbal or by minimal verbalization.

Regarding the socioeconomic level of families, nine (41\%) belonged to class A/B (high) and thirteen (59\%) to classes $C / D$ (medium-low), according to the socioeconomic classification of the ABEP ${ }^{23}$.

The mothers were on average 41 years and five months $(S D=7.9)$. Thirteen of them had college $(60 \%)$ and nine (40\%) high school.

As inclusion criteria, the following were considered: the diagnosis of ASD, the age group, the absence of oral communication or minimal verbalization, the child's attachment to educational institutions and the family's availability to participate in speech therapy sessions with a minimum adherence of $75 \%$.

Exclusion criteria were the presence of neurological alterations (structural and/or functional impairment of the Central Nervous System), malformations and/or known genetic syndromes, physical, auditory/visual and/or motor impairments.

To assess family engagement in the program, the PECS Executor Skills Assessment Protocol ${ }^{6}$ was used, which is composed of tasks that the adult needs to perform to ensure the successful implementation of each phase of the system, namely:

a. In Phase 1: the partner needs to tidy up the environment; to avoid verbal clues; to appropriately provoke; to use the open hand clue; to reinforce with social praise; to not insist on speech and to return the figure.

b. In Phase 2: the partner must provide the communicative folder; adapt the environment; appropriately provoke; increase the distance between them and the child; teach the child to go through the room, searching for the communicative partner, to teach the search for the communicative folder; gradually increase the distance; turn their back to the child; appropriately reinforce new behavior; eliminate clues from the coach; to not insist on speech and teach the child to move around the environment.

c) In Phase 3: the partner needs to adapt the environment; stimulate the child with two items; provide social reinforcements; reinforce appropriately with each item; use uninteresting items and target figures; conduct error correction procedures; move the figures in the folder; to not insist on speech; teach the child to discriminate between three and five items; use several target figures; teach the child to seek the figure inside the folder; to not insist on speech.

d. In Phase 4: the partner needs to start with "I want" in the sentence; wait for initiation; physically guide the child; decrease physical help; provide verbal compliments; teach how to assemble the strip; reinforce new behavior; reinforce with a tangible item; use physical support; use delay in strip reading; apply differential reinforcement if the child talks; avoid verbal clues; conduct error correction; organize the folder; to not insist on speech. In addition, the partner must identify reinforcers for the attributes; teach the construction of sentences; reinforce new behavior; teach attribute discrimination; conduct the verification of attributes; conduct error correction, teach several attributes by classes; teach several examples of each attribute.

e. Phase 5: the partner needs to use the hint delay; use differential reinforcement; reinforce new behavior; create multiple opportunities.

f. Phase 6: the partner must use the clue delay; continue creating opportunities for spontaneous requests; reinforce comments/requests; teach discrimination between questions; use error correction; teach "comment question"; create lessons for spontaneous commentary; decrease "comment question"; incorporate attributes; teach more comments.

In this study, the partner's skills were analyzed both qualitatively - as originally proposed by the PECS Training Manual ${ }^{7}$ using the criteria: subject approved (correct execution) or subject needs to improve (some failure in execution) - and quantitatively. For quantitative analysis, each skill received a score: 2 (correct execution); 1 (needs to improve); 0 (incomplete) and 00 (does not apply).

Therefore, parents had to actively participate in the entire program: they recorded everyday situations in the home through filming so that researchers could be sure of the proper use of the system, as well as the preparation of the environment and the construction of materials (example: select images, assemble cards), in each phase of the program (executor skills). 
In addition, family adherence was measured by frequency of sessions.

The program consisted of 24 individual speech therapy sessions with the active presence of the family member. Each session lasted 45 minutes and was held weekly. The number of weekly sessions was based on the experience of assistance developed in a teaching clinic within the SUS, in a previous study conducted by the same group of researchers, with the aim of corroborating this time interval as a sufficient period for implementing the system. All speech therapists involved were professionals trained and certified in PECS. All sessions were filmed so that the children's behaviors were recorded in the progress monitoring protocols at each stage, as proposed in the PECS ${ }^{6}$ Training Manual. The records were made by researchers who were not involved in the direct care of the children.
Initially, described analyses of the study variables were performed. To measure the correlations, the Spearman Correlation Test was used. A significance level of $0.05 \%$ was adopted.

\section{RESULTS}

In Figure 1, it is possible to analyze the percentage distribution of children's performance by stages of the Picture Exchange Communication System.

In Figure 2, it is possible to observe the average percentage of the skills of the executor, by phases of the Picture Exchange Communication System.

Table 1 shows the correlation between the skills of the performer and the number of sessions.

The adhesion of families to the program was, on average, $96 \%$.

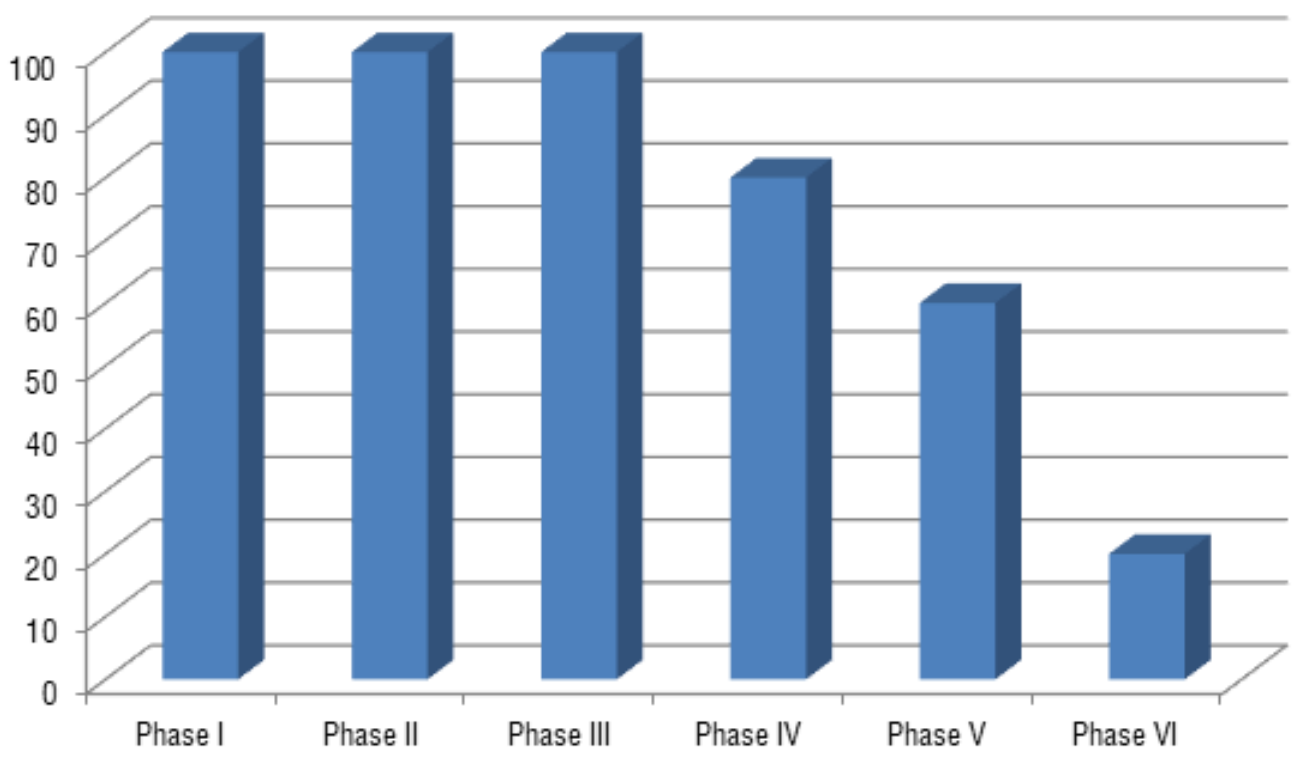

Figure 1. Children's performance by stages of the Picture Exchange Communication System 


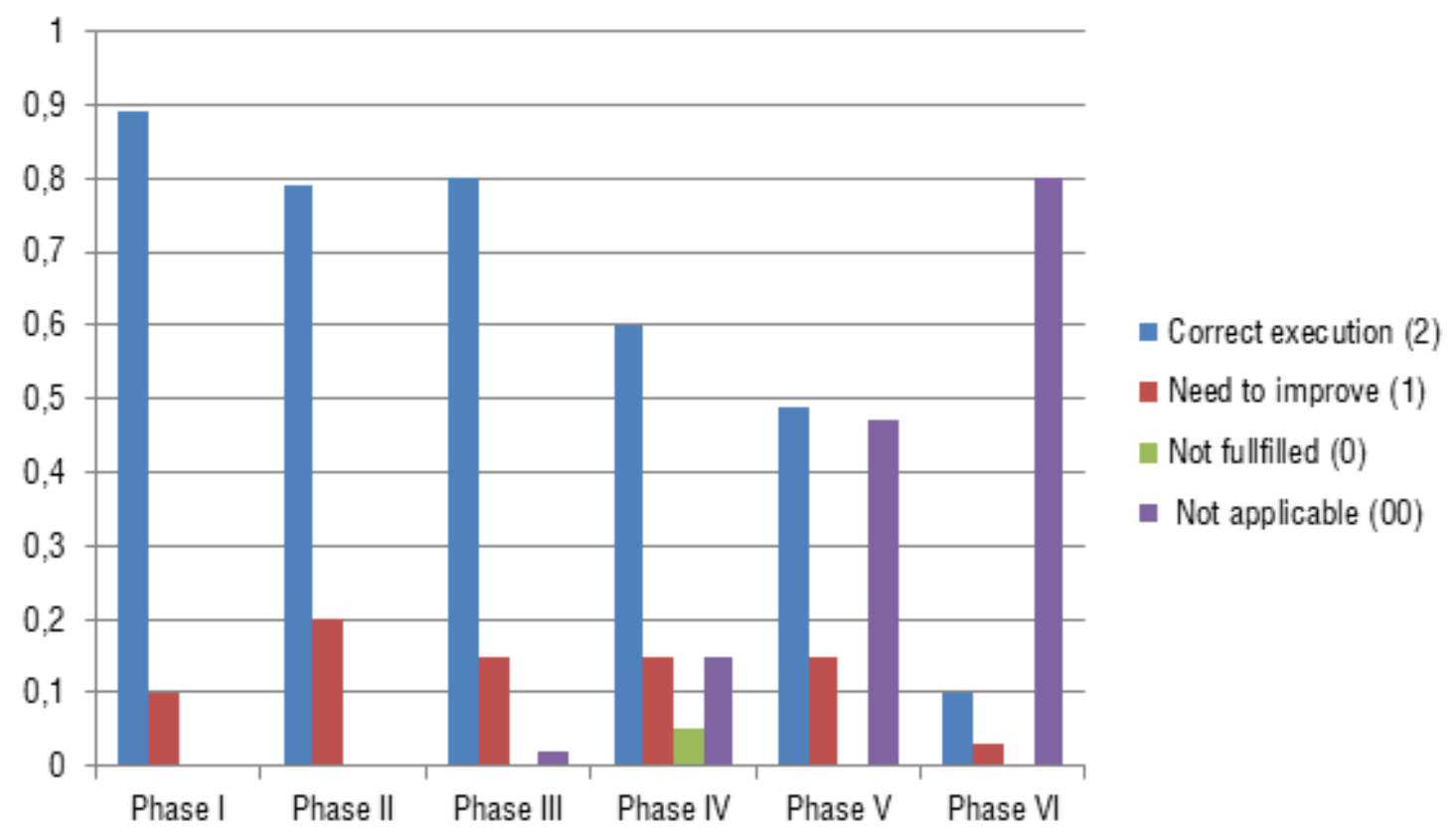

Figure 2. Average percentage of skills of the executor by phases of the Picture Exchange Communication System

Table 1. Correlation between executor skills and number of sessions of the Picture Exchange Communication System

\begin{tabular}{cccc}
\hline Phase/Sessions & Correlation coefficient & P value & N \\
\hline I (2 sessions) & 0.460 & $0.031^{*}$ & 22 \\
II (3 sessions) & -0.469 & $0.028^{*}$ & 22 \\
III (7 sessions) & 0.0465 & $0.029^{*}$ & 22 \\
IV (5 sessions) & 0.534 & $0.022^{*}$ & 18 \\
V (3 sessions) & -0.121 & 0.681 & 14 \\
VI (5 sessions) & -0.316 & 0.684 & 4 \\
\hline
\end{tabular}

Spearman's correlation test

\section{DISCUSSION}

In the analysis of the children's performance over the period of 24 sessions, it was noted that all children were able to discriminate and select the target card and deliver it to the interlocutor intentionally and autonomously. Therefore, there was no difficulty in reaching the first three phases of the system. About $82 \%$ of the children reached the next stage (stage IV) and started to build sentences using action verb cards and perceptual attributes, showing a significant increase in their lexical repertoire. Stage $V$ was reached by about $64 \%$ of children, as they became able to answer questions such as "what do you want?" using the cards. Only 19\% of the sample reached stage VI (Comments). This drop in performance between phases $\mathrm{V}$ and $\mathrm{VI}$ is most likely related to the complexity of the task and the resulting limitation of the child in understanding and performing the steps required in each of these phases ${ }^{6,24-30}$. Another factor, although less relevant, that may also have contributed to these results was the stipulated duration for the implementation of the program (24 sessions).

It was observed that the mothers correctly performed most of the tasks planned especially in the first three phases of the PECS. From phase IV on, it was noticed that the performance of the executor started to be affected by the performance of the children and by the complexity of the system. That is, by requiring an increase in the construction of grammatically more robust sentences, children began to show greater difficulty in performing the planned tasks and, therefore, this proportionally affected the opportunities for stimuli that were no longer created and offered by mothers. There is a clear decline in actions performed correctly by mothers and the proportional increase in "not applicable" actions from Phase IV to the end VI. 
In the analysis of the correlation between the skills of the performer and the number of sessions per phase of the PECS, it was noted that there was direct statistical significance in phases I, III and IV, that is, the strong commitment of the mothers to prepare adequate environments and several; and full of stimuli and opportunities for communicative exchange, in all sessions performed in these phases. On average, two sessions were used for phase I, seven sessions for phase III and five for phase IV.

Interestingly, the correlation, despite being significant, was indirect in phase II, that is, it can be observed that the mothers created opportune situations of communicative exchange, expanded the distance and offered different scenarios; and the children, in turn, responded promptly, needing only three sessions, on average, to show themselves able to pass the stage. This positive performance of children reflects, on the one hand, the successful implementation of phase I, and on the other hand, it shows how family engagement and empowerment can be considered essential factors for a successful therapeutic intervention ${ }^{11-21}$.

Although difficulties in implementing the PECS were observed, particularly in phases $\mathrm{V}$ and VI, the results obtained in this study allow us to state that family engagement was very relevant in the process of appropriation of the system by children, since adherence to the program was, on average, $96 \%^{11-21}$. And even though the children's performance in reaching the stages is directly independent of the executor's abilities, having a committed and available communication partner certainly allows communication exchanges to be more effective and efficient.

It is suggested that more studies on the use of alternative and augmentative communication systems such as PECS be conducted, especially in larger population samples, since the sample size of this study may have limited the statistical treatment of data. It is recommended to design more longitudinal studies and clinical trials so that it is possible to assess the positive impact of using PECS for longer periods of time.

\section{CONCLUSION}

Notably, the families' engagement was very significant for children to appropriate PECS in a relatively short time of exposure, reaching phases of discrimination and construction of sentences with cards.

\section{AKNOWLEDGEMENTS}

Special thanks to Soraia Vieira for the technical consultancy, to CNPq for the support in the form of Scientific Initiation Scholarship and Research Financing (421937/2018-1).

\section{REFERENCES}

1. American Psychiatric Association. Manual Diagnóstico e Estatístico de Transtornos Mentais DSM-5, Porto Alegre, Artmed, 2014.

2. Klin A, Jones W. An agenda for 21st century neurodevelopmental medicine: lessons from Autism. Rev Neurol. 2018;66(S01):S3-S15.

3. Moretto G, Ishihara MK, Ribeiro M, Caetano SC, Perissinoto J, Tamanaha AC. Interference of the communicative profile of children with autism spectrum disorders upon their mother's quality of life. Codas. 2020;32(6):e20190170.

4. Tamanaha AC, Chiari BM, Perissinoto J. The efficacy of the speech and language therapy in autism spectrum disorder. Rev CEFAC. 2015;17(2):552-8.

5. Ferreira $C$, Bevilacqua $M$, Ishihara $M$, Fiori $A$, Armonia A, Perissinoto $\mathrm{J}$ et al. Selection of words for implementation of the Picture Exchange Communication System - PECS in non-verbal autistic children. Codas. 2017;29(1):e20150285.

6. Bondy A, Frost L. Manual de treinamento do sistema de comunicação por troca de figuras. Newark: Pyramid, 2009.

7. Fortea-Sevilla MS, Escandell-Bermudez MO, Castro-Sanchez JJ, Martos-Pérez J. Early development of language in small children with autism spectrum disorder using alternative systems. Rev Neurol. 2015;60(Suppl 1):S31-5.

8. Ogletree BT, Morrow-Odorn KL, Westling D. Understanding the brain behavior relationship in persons with ASD: implications for PECS as a treatment choice. Dev Neurorehabil. 2015;18(2):88-96.

9. Schreibman L, Stahmer AC. A randomized trial comparison of the effects of verbal and pictorial naturalistic communication strategies on spoken language for young children with autism. J Autism Dev Disord. 2014;44(5):1244-51.

10. Ganz JB, Hong ER, Goodwyn F, Kite E, Gilliland W. Impact of PECS tablet computer app on receptive identification of pictures given a verbal stimulus. Dev Neurorehabil. 2015;18(2):82-7. 
11. Haney JL, Houser L, Cullen JA. Parental perceptions and child emotional and behavioral problems in Autism. J Autism Dev Disord. 2018;48(1):12-27

12. Emily G, Grace I. Family quality of life and Autism Spectrum Disorder: the role of child adaptative functions and behaviors problems. Autism Res. 2015;8(2):199-213.

13. Hong J, Fitzpatrick LB, Smith LE, Greenberg JS, Mailink MR. Factors associated with subjective quality of life of adults with autism spectrum disorder: self-report versus maternal reports. J Autism Dev Disord. 2016;46(4):1368-78.

14. Jones S, Bremer E, Lloyd M. Autism spectrum disorder: family quality of life while waiting for intervention services. Qual Life Res. 2017;26(2):331-42.

15. Øien R, Eisemann MR. Brief report: parentereported problems related to communication behavior and interests in children with autistic disorders and their impact on quality of life. J Aut Dev Disord. 2015;46(1):328-31.

16. Reed P, Sejunaite K, Osborne LA. Relating between self-reported health and stress in mother of children with Autism Spectrum Disorder. J Aut Dev Disord. 2016;46(3):934-41.

17. Siah PC, Tan SH. Relationships between sense of coherence, coping strategies and quality of life of parents of children with autism in Malaysia: a case study among chinese parents. Disability, CBR \& Inclusive Development. 2016;27(1):78.

18. Suzumura S. Quality of life in mothers of preeschoolers with high-functioning Pervasive Developmental Disorder. Pediatr Int. 2015;57(1):149-54.

19. Piovesan J, Scortegagna SA, Marchi ACB. Quality of life and depressive symptomatology in mothers of indivduals with autism. Psico-USF. 2015;20(3):505-15.

20. Ribeiro SB, Paula CS, Bordini D, Mari JJ, Caetano SC. Barriers to early identification of autism in Brazil. Rev. Bras. Psiquiatr. 2017;39(4):352-4.

21. Siller M, Hotez E, Swanson M, Delavenne A, Hutman T, Sigman M. Parent coaching increases the parents - capacity for reflection and self-evaluation: results from a clinical trial in autism. Attach Hum Dev. 2018;20(3):287-308.

22. Weschler D. WISC III Escala de inteligência para crianças. São Paulo, Casa Psicólogo, 2002.

23. Associação Brasileira de Empresas de Pesquisa. Critério de classificação econômica Brasil
[Homepage on the Internet]. São Paulo: ABEP; 2018. p. 1-6 [accessed on 2019 July 3]. Available at: www.abep.org

24. Doherty A, Bracken M, Gormley L. Teaching children with autism to initiate and respond to peer mands using Picture Exchange Communication System. Behav Anal Pract. 2018;11(4):279-88.

25. Landa R, Hanley GP. An evaluation of multiple schedule variations to reduce high rate request in the PECS. J Appl Behav Anal. 2016;49(2):388-93.

26. Agius MM, Vance M. A comparison of PECS and Ipad to teach requesting to preschollers with autistic spectrum disorders. Augument Altern Commun. 2016;32(1):58-68.

27. Thiemann-Bourque K, Brady N, McGuff S, Stump K, Naylor A. Picture Exchange Communication System and Pals: a peer-mediated augmentative and alternative communication intervention for minimally verbal preschoolers with autism. J Speech Lang Hear Res. 2016;59(5):1133-45.

28. Gilroy SP, Leader G, Mc Cleery JP. A pilot community-based randomized comparison of speech generating devices and the PECS for children diagnosed with Autism Spectrum Disorder. Autism Res. 2018;11(12):1701-11.

29. Brignell A, Chenausky KV, Song H, Zhu J, Suo C, Morgan AT. Communication interventions for Autism Spectrum Disorder in minimally verbal children. Cochrane Database Syst Rev. 2018;11(11):CD012324.

30. Jurgens A, Anderson A, Moore DW. Maintenance and generalization of skills acquired through PECS training: a long-term follow-up. Dev Neurorehabil. 2019;22(5):338-47. 